\title{
laaS Implementation of a Private Cloud using Open Source Technology
}

\author{
Rajat Kandpal \\ BTech CSE \\ College of Engineering Roorkee \\ Roorkee, India
}

\author{
Vishal Kumar \\ BTech CSE \\ College of Engineering Roorkee \\ Roorkee, India
}

\begin{abstract}
Cloud Computing is a field of IT that can revolutionize the traditional computing facilities. Cloud Computing gives a new view to our hardware and software facilities by making them elastic rather than scalable. The system can be scaled up or down according to a user's need. The use of open source technology is a turning point in providing cloud computing infrastructure to reduce the cost by a large magnitude. We can build our own private clouds and share resources viz. hardware and software without making any investment. The aim of this paper is to provide open source solution to build a private cloud. Iaas(Infrastructure as a service) implementation means to implement a private cloud wherein we can share hardware resources so as to reduce the cost of IT services of an organization.
\end{abstract}

\section{Keywords}

Cloud Computing, IAAS, Private Cloud, Open Source Technology, Eucalyptus, Hypervisors.

\section{INTRODUCTION}

Cloud Computing is a computing model, where resources such as computing power, storage, network and software are abstracted and provided as services on the internet in a remotely accessible fashion [5].In cloud computing everything is used as a service. The services can be billed as a pay-as-you-use basis. When we use open source technology we can cut short of payment of services but we must have a pool of resources to extract our computing requirements.

The main concept behind cloud computing is providing services. It provides various types of services, some of the important services are SaaS, PaaS and IaaS. Software as a service is a model of software deployment whereby a provider licenses an application to customers for use as a service on demand. Platform as a service generates all facilities required to support the complete cycle of construction and delivery of web-based applications wholly available in Internet without the need of downloading software or special installations by developers and finally Infrastructure as a service provides informatics resources, such as servers, connections, storage and other necessary tools to construct an application design prepared to meet different needs of multiple organizations, making it quick, easy and economically viable[8].

Cloud computing is mainly classified into three types based on the deployment model; Public cloud, Private cloud and Hybrid cloud. If the services are provided over the internet then it is public cloud or external cloud and if it is provided within an organization through intranet then it is named as private cloud or internal cloud and Hybrid cloud is an internal/external cloud which allows a public cloud to interact with the clients but keep their data secured within a private cloud [3].

In this paper we will build a Private cloud using Open Source Software Eucalyptus. The Eucalyptus package is bundled inside the UEC (Ubuntu Enterprise Cloud) which in turn comes inside Ubuntu Server 10.04. The private cloud implemented renders its services as Iaas.

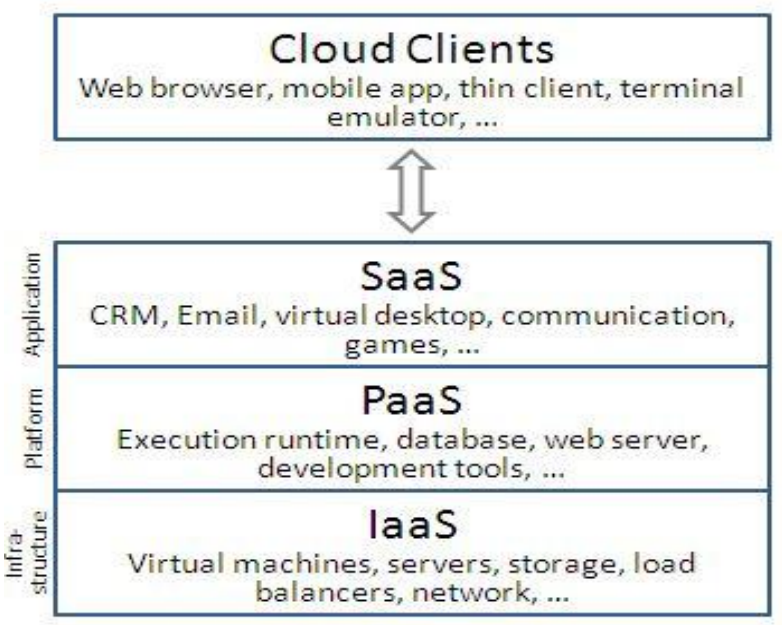

Fig.1: Types of Cloud Services[1]

\section{IAAS}

In the most basic cloud-service model, providers of IaaS offer computers - physical or (more often) virtual machines - and other resources. (A hypervisor, such as Xen or KVM, runs the virtual machines as guests. Pools of hypervisors within the cloud operational support-system can support large numbers of virtual machines and the ability to scale services up and down according to customers' varying requirements.) IaaS clouds often offer additional resources such as a virtualmachine disk image library, raw (block) and file-based storage, firewalls, load balancers, IP addresses, virtual local area networks (VLANs), and software bundles. IaaS-cloud providers supply these resources on-demand from their large pools installed in data centers. For wide-area connectivity, customers can use either the Internet or carrier clouds (dedicated virtual private networks).

To deploy their applications, cloud users install operatingsystem images and their application software on the cloud infrastructure. In this model, the cloud user patches and maintains the operating systems and the application software. Cloud providers typically bill IaaS services on a utility computing basis: cost reflects the amount of resources allocated and consumed. Examples of IaaS providers include: 
Amazon EC2, Azure Services Platform, Google Compute Engine, HP Cloud etc.[1]

\section{OPEN SOURCE}

Generically, open source refers to a program or a software in which the source code is available to the general public for use and/or modification from its original design free of charge, i.e., open to use and customize [12].

Open source software is by definition software for which users have access to the source code. This distinguishes it from the recent common practice of commercial software publishers by only releasing the binary executable versions of the software. Most open source software is also distributed at no cost with limited restrictions on how it can be used; hence the term free when used to describe open source carries two meanings: 1) free of cost and 2) free to do with the software as you wish (i.e., most importantly free to read and customize the code).[6]

Open source code is typically created as a collaborative effort in which programmers improve upon the code and share the changes within the community. Software is protected using the copyright system. Relying on the same protection as on books, music or film, the buyer of software is licensed the use of a copy of the product.

Open Source also allows free redistribution of software and allows us to customize it to meet our needs eg. UEC, EUCALYPTS, UBUNTU.

\section{UBUNTU ENETRPRISE CLOUD AND ITS ELEMENTS}

Ubuntu Enterprise Cloud, UEC for short, is a stack of applications from Canonical included with Ubuntu Server Edition. UEC includes Eucalyptus along with a number of other open source software. UEC makes it very easy to install and configure the cloud. Canonical also provides commercial technical support for UEC[5].

The figure shows a Private cloud implemented with Eucalyptus.

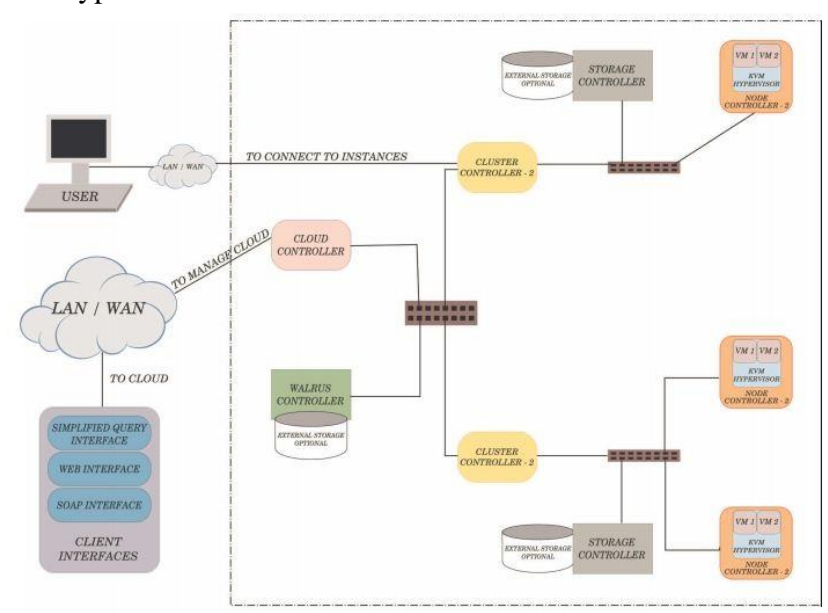

Fig.2: Private Cloud based on Eucalyptus[5]

\subsection{Eucalyptus}

Eucalyptus stands for "Elastic Utility Computing Architecture for Linking Your programs to Useful Systems". Eucalyptus is a research project under direction of Prof. Rich Wolski, Computer Science Department at the University of California, Santa Barbara, USA[7]. Eucalyptus is a software available under GPL that helps in creating and managing a private or even a publicly accessible cloud. It provides an EC2compatible cloud computing platform and S3-compatible cloud storage platform. The Eucalyptus Cloud maintains its compatibility with the Amazon EC2 cloud.

- Node Controller controls the execution, inspection,and terminating of VM instances on the host where it runs.

- Cluster Controller gathers information about and schedules VM execution on specific node controllers,as well as manages virtual instance network.

- Storage Controller (Walrus) is a put/get storage service that implements Amazon's S3 interface, providing a mechanism for storing and accessing virtual machine images and user data.

- Cloud Controller is the entry-point into the cloud for users and administrators. It queries node managers for information about resources, makes highlevel scheduling decisions, and implements them by making requests to cluster controllers[2]

A CLC manages the whole cloud and includes multiple CC's. There will be a WS3 attached to a CLC.A CC can contain multiple NC's and SC's. Ultimately the VM's will be running in the NC making use of its physical resources.

Eucalyptus is a platform for providing scalable internet service [2]. Eucalyptus allows to scale the cluster to multiple machines. Thus the resources can be easily added to the cloud eg. Node Controllers can be added to the cluster in the cloud.

\section{CONFIGURING AND INSTALLING THE PRIVATE CLOUD}

The UEC setup in Includes two servers (Server 1 and Server 2) which will run a Ubuntu 10.04 server version and the third system which will run a Lucid Desktop 64-bit version (Client 1)[5].

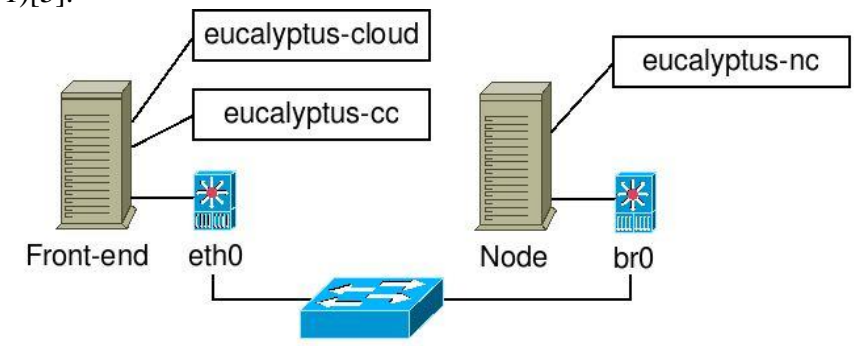

Fig.3: Private Cloud Setup Overview[13]

\subsection{Server 1 Install}

1. Insert ubuntu 10.04 server edition cd

2. Select "Install ubuntu enterprise cloud"

3. Configure the network: select "configure network manually"

address 192.168.6.22

gateway 192.168.0.1

netmask 255.255.255.0

name server 121.242.xxx.xxx

4. Host name for this system: host1 
5. Cloud controller address: leave it blank

6. Cloud installation mode:

Select following Cloud controller, Walrus storage service,

Cluster controller, Storage controller

7. Partition disks select "Guided-use entries disk and set up LVM"

8. Full name for new user and username for account : rajat

9. Select no automatic updates

10. Eucalyptus cluster name : cluster1

11. Pool of IP addresses that can be dynamically assigned as public IP's of virtual machines: 192.168.6.70-192.168.6.80

12. Install grub boot loader to master boot loader: yes[4]

Make sure you have latest version of eucalyptus

\$sudo apt-get upgrade eucalyptus

\subsection{Server 2 Install}

1. Insert ubuntu 10.04 server edition cd

2. Select "Install ubuntu enterprise cloud"

3. Configure the network: select "configure network manually"

address 192.168.6.23

gateway 192.168.6.22 (IP of cloud controller)

netmask 255.255.255.0

nameserver 121.242.xxx.xxx

Here Cloud controller is detected automatically

4. Host name for this system: host2

5. Cloud installation mode: Node controller

6. Partition disks select "Guided-use entire disk and set up LVM"

7. Full name for new user and username for account : rajat

8. Select no automatic updates

9. Install grub boot loader to master boot loader: yes[4]

\subsection{Client 1 Install}

The purpose of Client1 machine is to interact with the cloud setup, for bundling and registering new Eucalyptus Machine Images (EMI)

1.Boot the Desktop off the Ubuntu Desktop 10.04 CD and install. The Desktop will be on the enterprise network and will obtain an IP address through DHCP.

2. Install KVM to help us to install images on KVM platform and bundle them:

\$sudo apt-get install qemu_kvm

3.Install euca2ools to manage the cloud from it

\$sudo apt-get install euca2ools[5].
Note:1. we can also install KVM on Server1 to bundle our images.

2.The Client1 is connected to CLC.

\subsection{Exchange of Public SSH Keys}

Note: This is needed only when the node controller is not discovered in the cloud:

1.On node controller set a temporary password

\$ sudo passwd eucalyptus

2.On cloud controller

\$ sudo -u eucalyptus ssh-copy-id -i

/var/lib/eucalyptus/.ssh/id_rsa.pub eucalyptus@ 192.168.6.22

3.On node controller remove temporary password

\$ sudo passwd -d eucalyptus

\subsection{Get credentials}

1.On cloud controller: install credentials which consist of x.509

Certificates and environment variables.

Open web browser and enter following url:

https://192.168.6.22:8443/\#login and click on credentials tab

Download the credentials euca2-admin-x509.zip on cloud controller.

2.Alternatively, if you are on the command line of the Cloud Controller, you can run:

mkdir - $\sim$ /.euca

chmod $700 \sim /$.euca

cd $\sim /$ euca

sudo euca_conf --get-credentials mycreds.zip

unzip mycreds.zip

$\ln -\mathrm{s} \sim /$.euca/eucarc $\sim /$.eucarc

cd-

In the above steps we make a folder /.euca and download the credentials and unzip the credentials after changing their permissions.

To validate that everything is working correctly, get the local cluster availability details:

. /.euca/eucarceuca-describe-availability-zones verbose[13]

ra jatChost1:/\$ euca-describe-auailabilitu-zones verbose

AUAILABILITYZONE cluster1 192.168.6.22

AUAILABILITYZONE i- un types free / max cpu ram disk

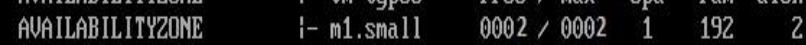

AUAILABILITYZONE i-c1.medium $0002 / 0002 \quad 1 \quad 256 \quad 5$

AUAILABILITYZONE $\quad$ - m1.large $0001 / 0001 \quad 2 \quad 512 \quad 10$

AUAILABILITYZONE i- $m 1$.xlarge $0001 / 0001221024 \quad 20$

AUAILABILITYZONE i-c1.xlarge $0000 / 0000 \quad 4 \quad 2048 \quad 20$

rajatChost1:/\$

Fig.4 : Screenshot of available resources. 
If you see the free/max VCPUs as 0 in the above list, it means the node did not get registered automatically. Please use the following on Server1 and approve when prompted to add 192.168.6.23 as the Node Controller:

\$sudo euca_conf --discover-nodes [7].

\subsection{Installing Images}

An Image in UEC is called a EMI (Eucalyptus Machine Image).A Eucalyptus Machine Image (EMI) is a combination of a virtual disk image(s), kernel and ramdisk images as well as an $\mathrm{xml}$ le containing metadata about the image. These images reside on WS3 and are used as templates for creating instances on UEC.

Each Linux EMI is a combination of the following:

•An XML le with a name like "jaunty.img.manifest.xml" with information about one or more hard disk images, a kernel image and a ramdisk image (id - emi-65440E7E)

-An XML le with a name like "vmlinuz-2.6.28-11server.manifest.xml" with information about the corresponding kernel image (id - eki-39FC1244)

-An XML le with a name like "initrd.img-2.6.28-11server.manifest.xml" with information about the corresponding ramdisk image (id - eri-71ED1322) site.[5]

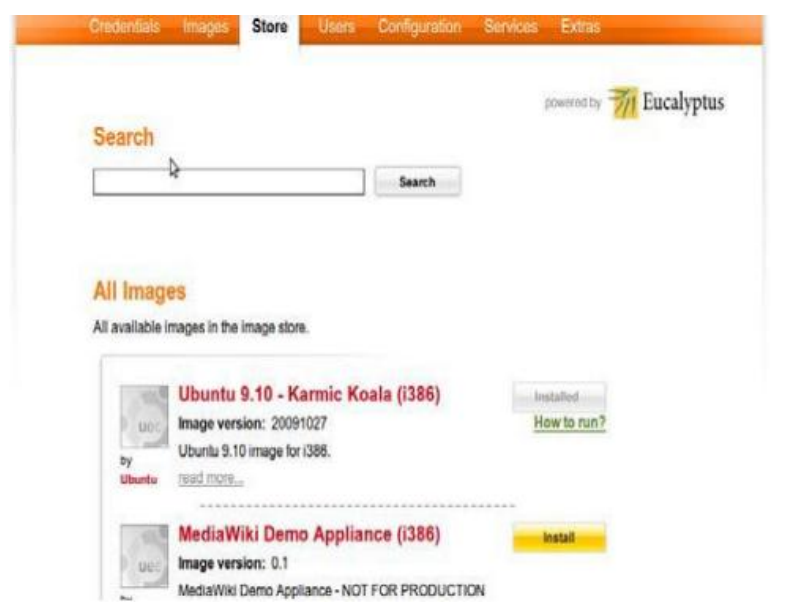

Fig.5 :Image of the Store

From the web interface of Eucalyptus, you can view a list of EMIs in the "Store" tab of the web UI. These are the EMIs listed from Canonical or partners. You can choose to download and install any of these images directly from Canonical's site.The images can also be customized ,bundled and uploaded to the cloud.

\subsubsection{Installing images in Server 1}

The Store tab provides list of images, also it can be installed using following commands:

Download images from Extras tab in UEC

1. Registering kernel image Execute the following commands to bundle and register the kernel image (vmlinuz-2.6.28-11server):

\$ euca-bundle-image -i vmlinuz-2.6.28-11-server --kernel true
\$ euca-upload-bundle -b mybucket -m /tmp/vmlinuz-2.6.2811-server.manifest.xml

\$euca-registermybucket/vmlinuz-2.6.28-11server.manifest.xml IMAGE eki-68101303

2.Registering ramdisk image Execute the following commands to bundle and register the ramdisk image (initrd.img-2.6.28-11-server):

\$ euca-bundle-image -i initrd.img-2.6.28-11-server

$\$$ euca-upload-bundle -b mybucket -m /tmp/initrd.img-2.6.2811-server.manifest.xml

\$ euca-register mybucket/initrd.img-2.6.28-11-

server.manifest.xml

IMAGE eri-A26613DD

3. Registering disk image Execute the following commands to bundle and register the disk image (centos.5-3.x86.img):

\$ euca-bundle-image -i centos.5-3.x86.img --kernel eki68101303 --ramdisk eri-A26613DD

$\$$ euca-upload-bundle -b mybucket - $\mathrm{m} / \mathrm{tmp} /$ centos.5-

3.x86.img.manifest.xml

\$ euca-register mybucket/centos.5-3.x86.img.manifest.xml

IMAGE emi-F62F1100

4. The new images that have been uploaded can be seen by using following command.

$\$$ euca-describe-images

\subsubsection{Install a Key Pair}

1. Create a new group called "wiki" and use it instead of the default group

\$ euca-add-group wiki -d wiki_demo_appliances

Also allow access to port 22 in instances:

\$ euca-authorize wiki -P tcp -p 22 -s 0.0.0.0/0

2. Build a keypair that will be injected into the instance(virtual

machine) allowing to access it via ssh.

\$ euca-add-keypair mykey > /.euca/mykey.priv

$\$$ chmod $0600 \sim /$ euca/mykey.priv [4]

\subsection{Running Instances}

1. Now we are finally ready to begin running instances. We'll start by creating an instance of our image and connections will be allowed on ports ssh and http:

\$ euca-run-instances $-\mathrm{g}$ wiki $-\mathrm{k}$ mykey $-\mathrm{t} \quad \mathrm{c} 1$. medium emiF62F1100

2. After issuing the "euca-run-instances" command to run an instance, we can track its progress from pending to running state by using the euca-describe-instances command. 


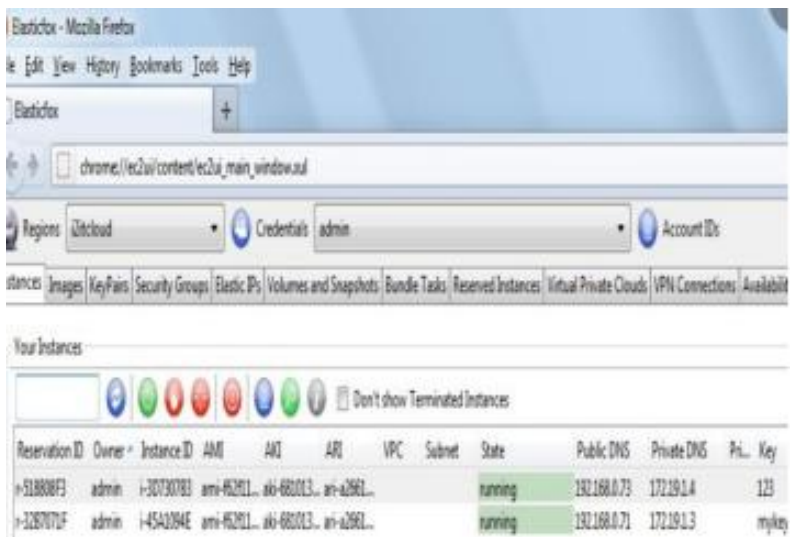

Fig.6: Running instances in hybridfox

At launch, Instances are in pending state after some time they are in running state at this state client can use the CentOS operating system. An instance to get terminated it first enters into shuttingdown state then it terminates[5]

\subsubsection{Hybridfox}

HybridFox (previously ElasticFox) is a web based GUI used to manage instances and other related aspects in the UEC. It is an extension for Mozilla's Firefox, and can be downloaded9

and installed easily. HybridFox 10 can connect to both public (Amazon) and private clouds (UEC) and provides compatibility between Amazon and UEC.

Hybridfox can perform all the functions that can be done by elasticfox, on the Eucalyptus Computing environment like Manage Images, Raise and Stop Instances, Manage Instances, Manage Elastic IPs, Manage Security Groups, Manage Key pairs and Manage Elastic Block Storage[11,16].

\subsection{Life Cycle of Instance}

When "euca-run-instances" command is invoked (or when run instance is chosen from Hybridfox/Elasticfox), the running process will be in a sequential manner as shown in Fig.7.Here are some few things that happen on various components of UEC:

1. Authentication/Authorization of the user request to ensure that we have permission to launch the instance

2. Identification of $\mathrm{CC}$ to take responsibility for deploying the instance and identification of the $\mathrm{NC}$ for running the instance.

3. Downloading the image from WS3 to NC (images are cached so that starting multiple instances of the same machine image downloads that image only once)[5].

\subsection{Scaling out the cloud}

To scale out the cloud we need to add NC(Node Controllers).We often get messages that the resources are not enough when we start an instance then there is a need to scale

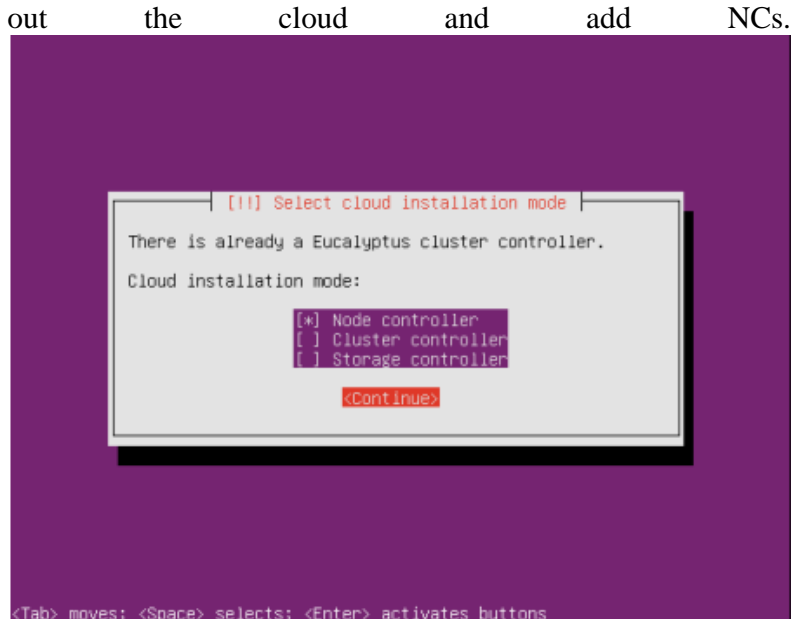

Fig.7: Screenshot of Installing Node controller

The NC are installed in the same way as done before and added to the cloud.

\section{FUTURE SCOPE}

The cloud can be integrated to other public clouds which gives rise to hybrid cloud. The integration also enhances the scope of cloud computing as we will be able to use resources of other organizations. They services can also be metered and made as pay-per-use basis. This kind of services are also called Saas(Software as a service).The cloud is dependent on internet and can be made to work in a lan.

Further work can be done to manage the private cloud with better resource management and improving the efficiency of the system.

\section{CONCLUSION}

Cloud computing is a colossal field, with which we can share our resources and couple them as in the private cloud. We have read many research papers, articles, web discussion about configuring a private cloud in the form of IaaS and managed to find a way of building it using open source softwares like UEC, eucalyptus. In the cloud we can share the hardware and other resources of an organization. The use of Open Source Technology makes our move very economical. The various softwares and operating systems used are open source and can be customized to meet our needs viz. Eucalyptus, Ubtuntu Server10.04 etc.

\section{REFERENCES}

[1] Cloud Computing,Wikipedia;en.wikipedia.org.

[2] Daniel Nurmi, Rich Wolski, Chris Grzegorczyk Graziano Obertelli, Sunil Soman, Lamia Youseff, Dmitrii Zagorodnov -The Eucalyptus Open-source Cloudcomputing System(2009)

[3] Judith H,Robin B,Marica K, And Dr.Fern H,Dummies.com,Comparing-Public-Private-andHybridcloud- computing. Wiley Publishing, Inc.2009.

[4] Louis Danuser (AT\&T Labs, Inc.) Deploying an Open Source Private Cloud On a Shoe String Budget Presentation,http://www.oscon.com/,2010 
[5] Eucalyptus Beginner's Guide-UEC Edition (Ubuntu Server 10.04-Lucid Lynx) v1.0 by Johnson D,Kiran Murari,Murthy Raju Suseendran RB,Yogesh Girikumar ,25 May 2010.

[6] The Open Source Software Development Phenomenon:An Analysis Based On Social Network Theory by Greg Madey,Vincent freeh,EighthAmericanConference on Information Systems (2002)

[7] Dr G.R.Karpagam and J.Parkavi, Setting Up of an Open Source Based Private Cloud,IJCSI,vol.8 ,Issue 3,May 2011.

[8] Glossary, (2010), MasterBase, www.en.masterbase.com/

[9] Simon Wardley, Etienne Goyer and Nickbarcet; Technical White Paper: Ubuntu Enterprise Cloud Architecture(2009).
[10] Simon Wardley,Etienne Goyer \& Nick Barcet,(2009),CANONICAL,Technical White Paper,Ubuntu Enterprise Cloud Architecture.

[11] Mitchell pronsc, Hybridfox: Elasticfox for Eucalyptus ,2009

[12] Open Source, http://www.webopedia.com.

[13] https://help.ubuntu.com/community/UEC

[14] Zoran Pantic and Muhammed Ali Babar, Installing and Scaling out Ubuntu Enterprise Cloud in Virtual Environment ;ISBN 978-87-7949-255-4 (2012).

[15] Installing the Eucalyptus Cloud/Cluster/Storage Node on Ubuntu Karmic 9.10 dustinkirkland, www.YouTube.com

[16] Ezhil Arasan Babaraj, (2009), Driving Technology Direction on Cloud Computing Platform, Blog post; Hybridfox: Cross of Elasticfox and Imagination, ezhil.syscon. com. 